\title{
A Reliable Method for Rhythm Analysis during Cardiopulmonary Resuscitation
}

\author{
U. Ayala, ${ }^{1}$ U. Irusta, ${ }^{1}$ J. Ruiz, ${ }^{1}$ T. Eftestøl, ${ }^{2}$ J. Kramer-Johansen, ${ }^{3}$ \\ F. Alonso-Atienza, ${ }^{4}$ E. Alonso, ${ }^{1}$ and D. González-Otero ${ }^{1}$ \\ ${ }^{1}$ Communications Engineering Department, University of the Basque Country UPV/EHU, Alameda Urquijo S/N, \\ 48013 Bilbao, Spain \\ ${ }^{2}$ Department of Electrical Engineering and Computer Science, Faculty of Science and Technology, \\ University of Stavanger, 4036 Stavanger, Norway \\ ${ }^{3}$ Norwegian Centre for Prehospital Emergency Care (NAKOS), Oslo University Hospital and University of Oslo, 0424 Oslo, Norway \\ ${ }^{4}$ Department of Signal Theory and Communications, University Rey Juan Carlos, Camino del Molino S/N, 28943 Madrid, Spain
}

Correspondence should be addressed to U. Irusta; unai.irusta@ehu.es

Received 10 February 2014; Revised 26 March 2014; Accepted 28 March 2014; Published 7 May 2014

Academic Editor: Yongqin Li

Copyright ( 2014 U. Ayala et al. This is an open access article distributed under the Creative Commons Attribution License, which permits unrestricted use, distribution, and reproduction in any medium, provided the original work is properly cited.

\begin{abstract}
Interruptions in cardiopulmonary resuscitation (CPR) compromise defibrillation success. However, CPR must be interrupted to analyze the rhythm because although current methods for rhythm analysis during CPR have high sensitivity for shockable rhythms, the specificity for nonshockable rhythms is still too low. This paper introduces a new approach to rhythm analysis during CPR that combines two strategies: a state-of-the-art CPR artifact suppression filter and a shock advice algorithm (SAA) designed to optimally classify the filtered signal. Emphasis is on designing an algorithm with high specificity. The SAA includes a detector for low electrical activity rhythms to increase the specificity, and a shock/no-shock decision algorithm based on a support vector machine classifier using slope and frequency features. For this study, 1185 shockable and 6482 nonshockable 9-s segments corrupted by CPR artifacts were obtained from 247 patients suffering out-of-hospital cardiac arrest. The segments were split into a training and a test set. For the test set, the sensitivity and specificity for rhythm analysis during CPR were $91.0 \%$ and $96.6 \%$, respectively. This new approach shows an important increase in specificity without compromising the sensitivity when compared to previous studies.
\end{abstract}

\section{Introduction}

Out-of-hospital cardiac arrest (OHCA) is a leading cause of mortality in the industrialized world, with an estimated annual incidence between 28 and 55 cases per 100,000 person-years [1]. Early cardiopulmonary resuscitation (CPR) and early defibrillation are the key interventions for survival after cardiac arrest [2]. Defibrillation may be administered by an automated external defibrillator (AED), which incorporates a shock advice algorithm (SAA) that analyzes the ECG to detect shockable rhythms. Current CPR guidelines emphasize the importance of high quality CPR with minimal interruptions in chest compressions (CCs) [3]. However, CPR must be interrupted for a reliable rhythm analysis because CCs produce artifacts in the ECG. These interruptions adversely affect the probability of defibrillation success and subsequent survival [4]. Currently, CPR is interrupted every 2 minutes for rhythm reassessment on an artifact-free ECG.

Although different approaches to rhythm analysis during CPR have been explored, for instance, algorithms that directly diagnose the ECG corrupt with CPR artifacts $[5,6]$, filtering the CPR artifact has been a major approach (see [7] for a comprehensive review). The time-varying characteristics of the CPR artifact and its spectral overlap with both shockable and nonshockable cardiac arrest rhythms mandate the use of adaptive filters [8], which use reference signals to model the CPR artifact. Over the years, many solutions have been proposed, including Wiener filters [9], Matching Pursuit Algorithms [10], Recursive Least Squares [11], least mean squares (LMS) [12], or Kalman filters [13, 14]. Adaptive solutions using exclusively the ECG have also been explored $[15,16]$, but the results were poorer. To evaluate 
the performance of these methods, researchers first filtered the CPR artifact and then analyzed the rhythm using a SAA to obtain the sensitivity and specificity of the method, that is, the proportion of correctly diagnosed shockable and nonshockable rhythms, respectively. However, the SAAs used were originally designed to analyze artifact-free ECG instead of the ECG after filtering.

Currently rhythm analysis during CPR is not possible [17]. Most methods have sensitivity above 90\%, the minimum value recommended by the American Heart Association (AHA) for SAA on artifact-free ECG [18]. However, specificity rarely exceeds $85 \%$, well below the $95 \%$ value recommended by the AHA. A low specificity would result in a large number of false shock diagnoses during CPR, which would unnecessarily increase the number of interruptions in CPR. Overall, the main cause of the low specificity is filtering residuals in nonshockable rhythms. These residuals frequently resemble a disorganized rhythm [10, 12] and are often misdiagnosed as shockable by SAAs designed to analyze artifact-free ECG. This problem is more prominent when the electrical activity of the underlying heart rhythm is low, particularly for asystole (ASY) $[14,16]$, because filtering residuals may have amplitudes comparable or larger than those of the underlying ECG.

In this study we explore the possibility of combining adaptive filtering techniques with a SAA designed to optimally classify the rhythm after filtering. The aim is to improve the accuracy of current approaches and in particular to overcome the low specificity. When compared to previous studies, our results showed an increased specificity without compromising the sensitivity, for a comprehensive dataset of OHCA rhythms.

\section{Materials and Methods}

2.1. Data Collection. The data for this study were extracted from a large prospective study of OHCA conducted between 2002 and 2004 in three European sites [21, 22]. CPR was delivered by trained ambulance personnel in adherence to the 2000 resuscitation guidelines. Episodes were recorded using modified Laerdal Heartstart 4000 defibrillators (4000SP) and an external CPR assist pad to acquire additional reference signals. All signals were acquired with a $500 \mathrm{~Hz}$ sampling rate. The initial rhythm and all subsequent changes in rhythm were annotated by consensus of an experienced anesthesiologist and a biomedical engineer, both specialized in resuscitation $[21,22]$. Rhythm annotations comprised five types (see [21] for further details): VF and fast ventricular tachycardia (VT) in the shockable category and ASY, pulseless electrical activity (PEA), and pulse-generating rhythm (PR) in the nonshockable category. Intervals with chest compressions were annotated using the compression depth (CD) obtained from the CPR assist pad.

For this study specific records containing the ECG and CD signals were automatically extracted from the original episodes. First rhythm transitions were identified using the original annotations, and then for each interval without rhythm transitions at most one record was extracted to avoid bias due to data selection. Records were extracted if the following criteria were met: duration of more than 20-s, ongoing CCs, and the same rhythm annotation before and after CCs. Following the AHA statement the records were grouped into a shockable and a nonshockable category. The amplitude thresholds adopted for coarse VF and ASY are those accepted in the literature on SAAs $[6,18]$. The following criteria and rhythm definitions were checked in the clean intervals before and after CCs.

Shockable Rhythms. This category includes fast VT, with rate above 150 beats per minute (bpm), and coarse VF. Coarse VF was defined as VF with peak-to-peak amplitude above $200 \mu \mathrm{V}$ and a fibrillation frequency above $2 \mathrm{~Hz}$.

Nonshockable Rhythms. These rhythms were further divided into the following:

(i) organized rhythms (ORG): all nonshockable rhythms except ASY (PEA and PR),

(ii) asystole (ASY): rhythms with peak-to-peak amplitudes below $100 \mu \mathrm{V}$ for at least 2-s.

All signals were resampled to $f_{s}=250 \mathrm{~Hz}$, a sampling rate similar to that used by commercial AEDs. In what follows, the sample index and time variables are related by $t=n \cdot T_{s}\left(T_{s}=\right.$ $1 / f_{s}$ ). The ECG was band limited to $0.5-30 \mathrm{~Hz}$ (order 10 Butterworth filter), a typical ECG monitor bandwidth used in AEDs $[5,6]$, which removes base line wander and high frequency noise.

Following standard practice in SAA design, the rhythm analysis method was designed to analyze three consecutive $3 \mathrm{~s}$ windows, so it gives a diagnosis every $9 \mathrm{~s}[23,24]$. A $3 \mathrm{~s}$ window is sufficient to characterize the rhythm in terms of rate, stability, and morphology and to make a shock (Sh) or no-shock (NSh) decision [23]. SAA algorithms combine several consecutive diagnoses to avoid errors due to rhythm transitions and to avoid shock diagnoses for short bursts of nonsustained VT. Therefore, each record was divided into nonoverlapping $9 \mathrm{~s}$ segments. The $9 \mathrm{~s}$ segments were randomly split into two separate sets, one to train the algorithm and an independent set to test the algorithm, as required by the AHA statement. In addition we made sure that the patients on both sets were different (AHA statement) and that the distribution of rhythm types was similar in both sets.

2.2. Rhythm Analysis Method. The block diagram of the rhythm analysis method is shown in Figure 1. First, a CPR artifact suppression filter estimates the underlying rhythm, that is, the filtered ECG signal, $s_{\text {filt }}$. Then, a SAA diagnoses every $3 \mathrm{~s}$ window of the filtered signal. The SAA is designed to optimally classify the filtered signal and is further composed of two sequential subalgorithms: (1) a detector of rhythms with low electrical activity (LEA), that is, nonshockable rhythms without distinguishable QRS complexes such as ASY or idioventricular rhythms, and (2) a Sh/NSh algorithm that classifies windows with electrical activity as shockable or nonshockable. 


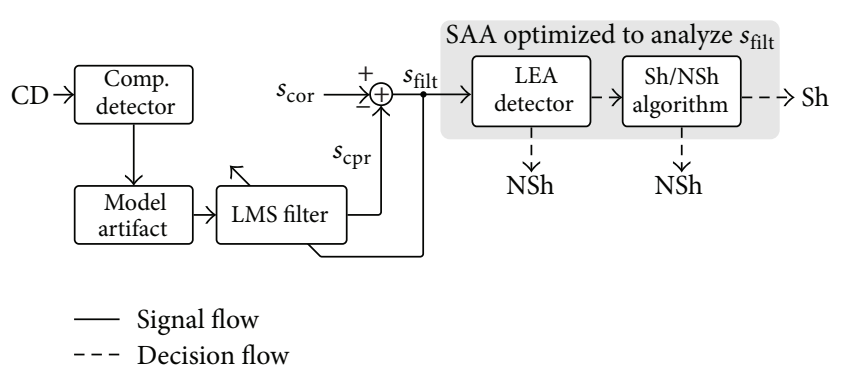

FIGURE 1: Block diagram of the new approach to rhythm analysis during CPR in which an adaptive filter (LMS filter based on the CD signal) is used in combination with a SAA designed to optimally classify the filtered signal, $s_{\text {filt }}$.
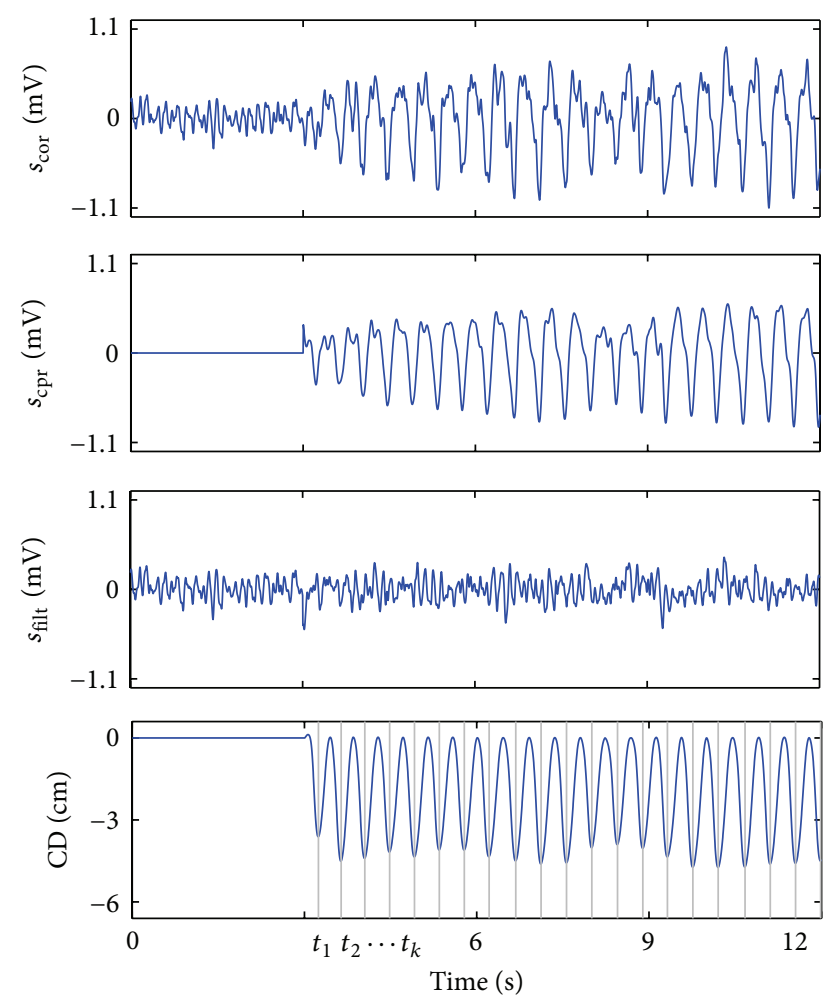

FIGURE 2: Filtering example of a 12-s segment. In the first $3 \mathrm{~s}$ there is no artifact and the underlying VF is visible. The filter estimates the artifact, $s_{\mathrm{cpr}}$, using information derived from the CC marks, indicated by vertical lines in CD $\left(t_{k}\right.$ instants).

2.3. Chest Compression Artifact Filter. CPR artifacts were suppressed using a state-of-the-art method based on an LMS filter [12]. In this method, CC artifacts are modeled as a quasiperiodic interference with a time-varying fundamental frequency, $f_{o}(n)$, which is the instantaneous frequency of the CCs. This frequency is derived from the $t_{k}$ instants, the CC marks shown in Figure 2. The LMS algorithm adaptively estimates the time-varying amplitudes, $c_{k}(n)$, and phases, $\phi_{k}(n)$, of the first 5 harmonics of the artifact by fitting the following model:

$$
\begin{gathered}
s_{\mathrm{cpr}}(n)=\sum_{k=1}^{5} c_{k}(n) \cos \left(k \cdot 2 \pi f_{o}(n) \cdot n+\phi_{k}(n)\right), \\
f_{o}(n)=\frac{1}{t_{k}-t_{k-1}} \text { for } t_{k-1} \leq n T_{s}<t_{k}
\end{gathered}
$$

In summary, the LMS algorithm dynamically estimates the CPR artifact by adaptively estimating its harmonic content. For this study, we used the optimal values of the filter parameters as described in $[12,19]$. As shown in Figure 1, the filtered signal was obtained by subtracting the estimated CPR artifact from the corrupted ECG. Figure 2 shows those signals for a 12-s segment with an underlying VF rhythm.

2.4. Shock Advice Algorithm. The SAA consists of a LEA detector followed by the Sh/NSh algorithm. The LEA detector identifies LEA windows as nonshockable; the rest of the windows are further processed by the Sh/NSh algorithm for a definitive diagnosis.

2.4.1. LEA Rhythm Detector. Some nonshockable rhythms (ASY, bradyarrhythmias or idioventricular rhythms) may not present QRS complexes in a $3 \mathrm{~s}$ analysis window. In these cases, filtering the CC artifact results in $s_{\text {filt }}$ with low amplitudes and short intervals in which the electrical activity is very low (see Figure 3(a)). To further improve LEA detection $s_{\text {filt }}$ was high pass filtered with a $2.5 \mathrm{~Hz}$ cut-off frequency using an order 5 Butterworth filter, which removed slow fluctuations of the ECG in LEA rhythms but preserved most frequency components of VF, as shown in Figure 3. The resulting signal, $s_{\mathrm{LEA}}$, was used to obtain the following two features:

(i) $P_{\text {LEA }}$ : energy of $s_{\text {LEA }}$ in the 3 s window:

$$
P_{\mathrm{LEA}}=\sum_{n} s_{\mathrm{LEA}}^{2}(n)
$$

(ii) $L_{\text {min }}:$ minimum of the curve lengths of $s_{\mathrm{LEA}}$ for nonoverlapping 0.5-s intervals, which measures the minimum electrical activity in 0.5 -s intervals. In discrete form, the curve length of the $k$ th subinterval is [25]

$$
L_{k}=\sum_{n=k f_{s} / 2+1}^{(k+1) f_{s} / 2} \sqrt{\Delta s_{\mathrm{LEA}}^{2}(n)+T_{s}^{2}} \text {, }
$$

where $\Delta s_{\text {LEA }}$ is the first difference of $s_{\text {LEA }}$.

LEA rhythms have smaller values of $P_{\mathrm{LEA}}$ and $L_{\text {min }}$ than shockable rhythms, as shown in Figure 3. This block was designed as a detector; that is, it gives a NSh diagnosis if a LEA rhythm is detected; otherwise the window is further processed by the Sh/NSh algorithm. 

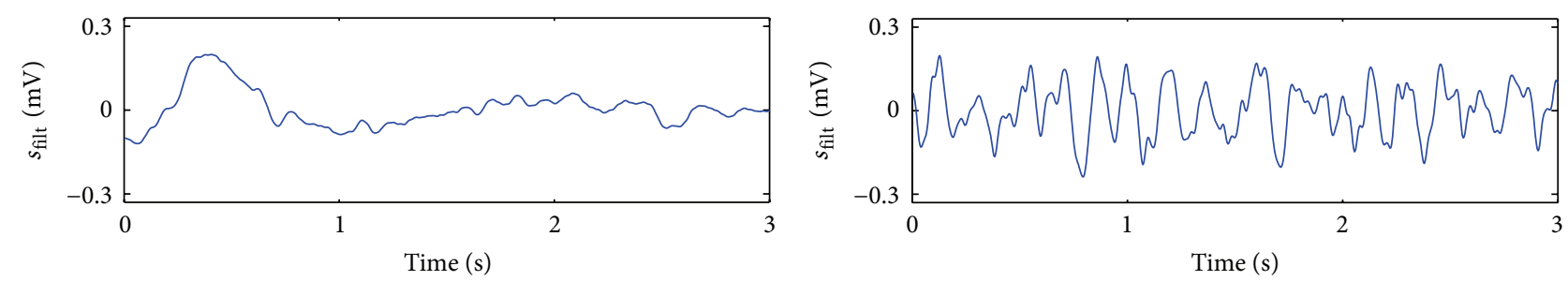

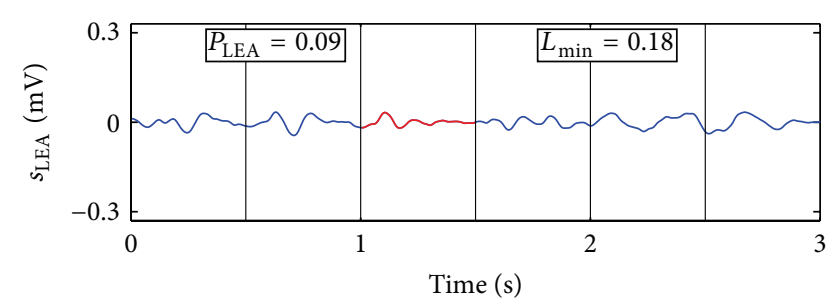

(a) ASY

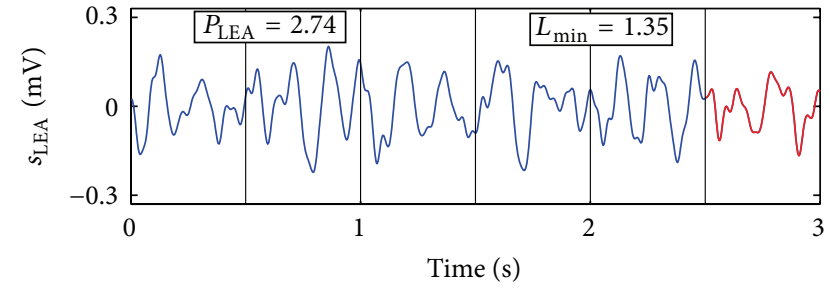

(b) $\mathrm{VF}$

FIGURE 3: Examples of a LEA rhythm (a) and a VF (b) window after filtering the CC artifact, $s_{\text {filt }}$, and preprocessed to suppress components under $2.5 \mathrm{~Hz}, s_{\mathrm{LEA}}$. Vertical lines separate the 0.5 subintervals, and the one with lowest activity $\left(L_{\text {min }}\right)$ is shown in red.

2.4.2. Sh/NSh Algorithm. During resuscitation, ORG rhythms with electrical activity may be very different in terms of rate, QRS width, or QRS morphology. Furthermore, even after CPR artifact suppression, rhythms may present important filtering residuals that may resemble VF. Four features derived from the frequency domain and slope analyses were defined. For rhythms with electrical activity, these features emphasize the differences between nonshockable (with QRS complexes) and shockable (without QRS complexes) rhythms.

2.4.3. Slope Analysis Features. QRS complexes were enhanced in $s_{\text {filt }}$ by computing the moving average of the square of its first difference (its slope):

$$
d_{\text {filt }}(n)=\frac{1}{N} \sum_{k=0}^{N-1}\left(s_{\text {filt }}(n-k)-s_{\text {filt }}(n-k-1)\right)^{2},
$$

where $N$ corresponds to the number of samples in a $100 \mathrm{~ms}$ interval. Then, $d_{\text {filt }}$ was divided by its maximum value in the

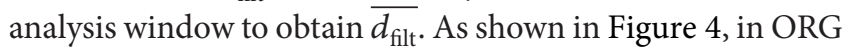
rhythms $\overline{d_{\text {filt }}}$ is large only around QRS complexes and very small otherwise, whereas in VF the values of $\overline{d_{\text {filt }}}$ are more evenly distributed and present many peaks. Two features were defined to measure these differences:

(i) $b S$ : slope baseline, a measure of how concentrated slope values are around small values (baseline), computed as the 10th percentile of $\overline{d_{\text {filt}}}$,

(ii) $n P$ : number of peaks above a fixed threshold in $\overline{d_{\text {filt }}}$.

Shockable rhythms will present larger values of $b S$ and $n P$ as shown in Figure 4.

2.4.4. Frequency Domain Features. For the frequency analysis, a Hamming window was applied to $s_{\text {filt }}$ and its zero padded 1024-point FFT was computed. The power spectral density was estimated as the square of the magnitude of the FFT and normalized to total power of one to give $P_{\mathrm{ss}}(f)$. As shown in Figure 5, VF concentrates most of its power around the fibrillation frequency, whereas ORG rhythms may have important power content at higher frequencies, on the harmonics of the heart rate. Two discrimination features were defined, with limiting frequencies in line with the characteristics of human VF $[26,27]$ :

(i) $P_{\mathrm{fib}}$ : power proportion around the VF-fibrillation band $(2.5-7.5 \mathrm{~Hz})$,

(ii) $P_{h}$ : power proportion in the high spectral bands (above $12 \mathrm{~Hz}$ ).

Shockable rhythms have larger values of $P_{\text {fib }}$ but lower values of $P_{h}$ (see Figure 5).

2.4.5. Support Vector Machine (SVM) Classifier. The Sh/NSh algorithm classified windows using a SVM with a Gaussian kernel [28]. First, features were standardized to zero mean and unit variance using the data in the training set. These $\mathbf{x}_{i}$ vectors of four normalized features were arranged as $\left\{\left(\mathbf{x}_{1}, y_{1}\right), \ldots,\left(\mathbf{x}_{n}, y_{n}\right)\right\} \in \mathbb{R}^{4} \times\{ \pm 1\}$, where $y_{i}=1$ for shockable and $y_{i}=-1$ for nonshockable windows. After training, the discriminant function for a window with feature vector $\mathbf{x}$ is

$$
f(\mathbf{x})=\sum_{i=1}^{N_{s}} \alpha_{i} y_{i} \exp \left(-\gamma\left\|\mathbf{x}-\mathbf{x}_{i}\right\|^{2}\right)+b
$$

where $\mathbf{x}_{i}$ are the support vectors, $N_{s}$ is the number of support vectors, and $\alpha_{i}$ and $b$ are coefficients estimated during training. Windows were classified as shockable for $f(\mathbf{x})>0$ or nonshockable for $f(\mathbf{x}) \leq 0$. Selecting an optimal SVM model for the classification problem involves selecting two parameters: $C$ and $\gamma$. The width of the Gaussian kernel, $\gamma$, determines the flexibility of the decision boundary [28]. The soft margin parameter, $C$, is used exclusively in the optimization process and is a tradeoff between classification 

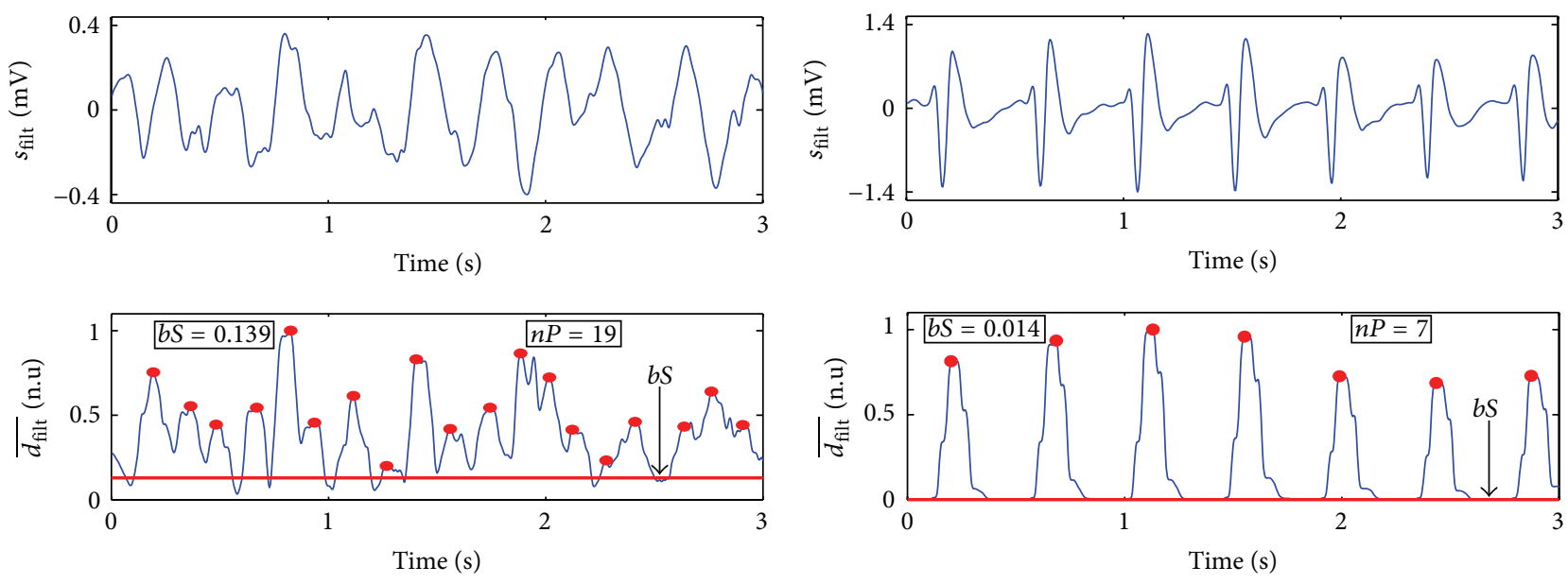

(a)

(b)

FIGURE 4: Example of the slope analysis for VF (a) and an ORG (b) window. During VF the slope, $\overline{d_{\text {filt }}}$, is irregular with many peaks, whereas ORG rhythms are regular with fewer peaks and concentrate most $\overline{d_{\text {filt }}}$ values around the baseline.
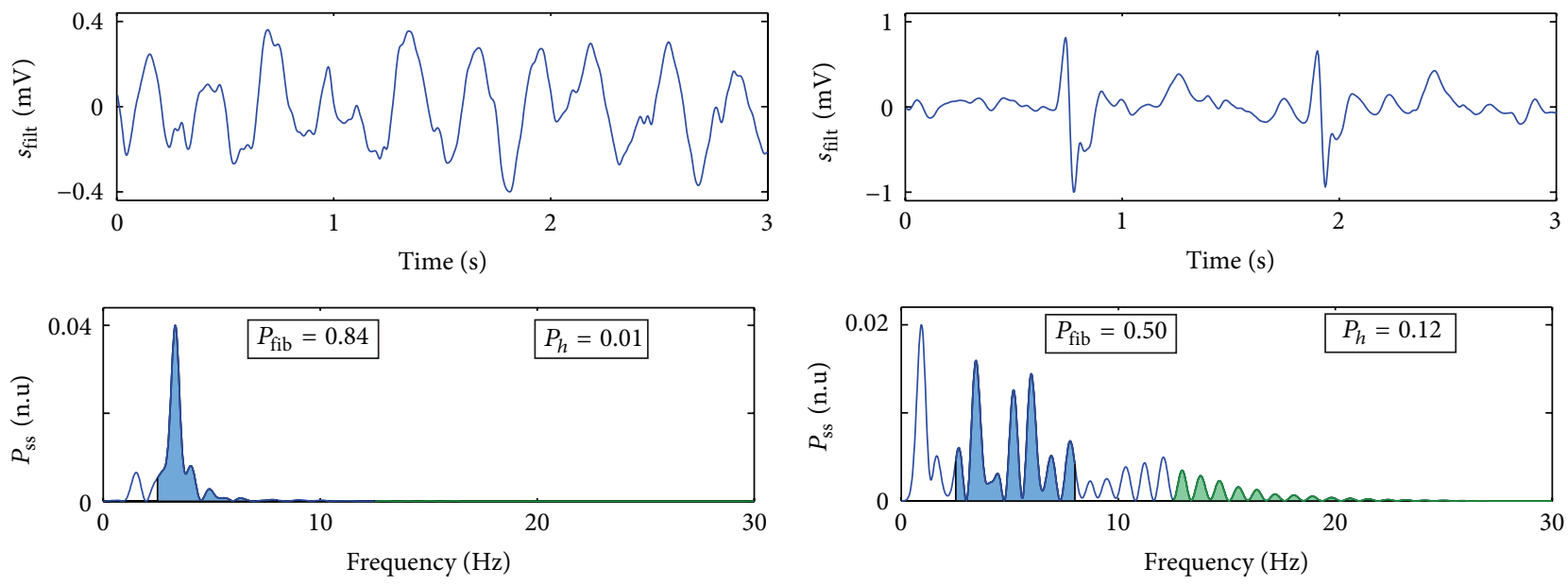

(a) VF

(b) ORG

FIGURE 5: Example of the frequency domain analysis for VF (a) and an ORG (b) window. VF concentrates most of its power around the fibrillation band (blue). ORG rhythms have a spectrum with many harmonics of the heart rate and thus larger $P_{h}$ (in green).

errors in training data and separating the rest of the training data with maximum margin [28].

2.5. Data Analysis and Algorithm Optimization. The rate and depth characteristics of CPR in our data were analyzed for each $9 \mathrm{~s}$ segment. The distributions for rate and depth did not pass the Kolmogorov-Smirnov test for normality and are reported as median and 5-95 percentiles.

For each discrimination feature of the SAA, statistical differences in medians between the targeted classification groups of each subalgorithm were measured using the MannWhitney $U$ test. The optimization process was carried out for the $3 \mathrm{~s}$ windows of the training set in two sequential steps.

(1) LEA Detector. ASY and shockable rhythms were used. The detection thresholds were determined through a greedy search on the two-dimensional feature space to jointly maximize the number of detected ASY and minimize the number of shockable windows incorrectly detected as nonshockable. An additional restriction was imposed: at maximum $5 \%$ of shockable windows could be incorrectly classified.

(2) Sh/NSh Algorithm. Shockable and ORG windows not detected as NSh by the LEA detector were used to optimize the SVM classifier. To avoid overfitting the SVM to the training set, $C$ and $\gamma$ were selected using 5-fold crossvalidation [29] to optimize the balanced error rate (BER):

$$
\mathrm{BER}=1-\frac{1}{2}(\mathrm{TPR}+\mathrm{TNR})
$$

where the true positive rate (TPR) and the true negative rate (TNR) are the capacity of the SVM 
TABLE 1: Number of segments (patients in parenthesis) and characteristics of the CC rate and depth for the training and test datasets. Values for CC rate and depth are presented as median with 5-95 percentiles in parenthesis.

\begin{tabular}{|c|c|c|c|c|c|c|}
\hline \multirow{2}{*}{ Rhythm type } & \multicolumn{3}{|c|}{ Training } & \multicolumn{3}{|c|}{ Testing } \\
\hline & 9-s seg. & Rate (cpm) & Depth (mm) & 9-s seg. & Rate (cpm) & Depth (mm) \\
\hline Shockable & $563(35)$ & $116(92-143)$ & $38(25-47)$ & $622(34)$ & $113(89-157)$ & $35(20-50)$ \\
\hline Nonshockable & $3132(110)$ & $116(88-155)$ & $36(20-51)$ & 3350 (109) & $116(84-159)$ & $35(21-57)$ \\
\hline AS & $1173(66)$ & $118(92-164)$ & $35(18-52)$ & $1309(60)$ & $117(89-151)$ & $34(21-52)$ \\
\hline ORG & $1959(66)$ & $114(86-149)$ & $37(23-51)$ & $2041(76)$ & $116(79-164)$ & $35(21-59)$ \\
\hline Total & $3695(123)$ & $116(89-151)$ & $36(21-51)$ & $3972(124)$ & $116(86-159)$ & $35(21-58)$ \\
\hline
\end{tabular}

classifier to detect shockable and ORG windows, respectively. Weights were assigned to each class to resolve the unbalance in the number of instances per class [28]. The best SVMs using one, two, or three features were compared to the optimal four-feature SVM using McNemar's test.

The performance of the algorithm was measured in the test set in terms of sensitivity and specificity. Since both $3 \mathrm{~s}$ windows and $9 \mathrm{~s}$ segments correspond to consecutive analyses within a record, the sensitivities, specificities, and their 90\% low one-sided confidence intervals (CI) were adjusted for clustering (longitudinal data) within each record, using a longitudinal logistic model fit by generalized estimating equations (GEE) [30, 31]. The analysis was carried out in $R$ using the geepack library [32]. Finally, the algorithm was programmed in MATLAB R2013a (Mathworks Inc.) for Windows and processing time performance tests were carried out on a $2.9 \mathrm{GHz}$ Intel i7 with $4 \mathrm{~GB}$ of RAM.

\section{Results}

3.1. Database Description. Our data comprise $76679 \mathrm{~s}$ segments within 1396 records extracted from 247 OHCA patient episodes. The median number of $9 \mathrm{~s}$ segments per record was 3 (1-19, range 1-44). Table 1 shows the number of 9 s segments and the rate and depth of CCs for those segments in the training and test sets. The median CC rate and depth were 116 (88-156) compressions per minute (cpm) and $36(21-53)$ $\mathrm{mm}$, respectively.

\subsection{Shock Advice Algorithm}

3.2.1. Training. Figures 6(a) and 6(b) show the values of $P_{\text {LEA }}$ and $L_{\min }$ for the ASY and shockable rhythms which presented significant differences between the two groups $(P<0.001)$. The optimal detection thresholds of the LEA detector were

$$
P_{\text {LEA }}<0.44 \text { or } L_{\text {min }}<0.63 \text {. }
$$

The LEA detector identified as NSh $72.1 \%$ of the ASY (true detections) and $0.9 \%$ of the shockable (false detections) windows. In addition, $38.8 \%$ of the ORG windows were correctly identified as NSh; these rhythms corresponded to very low rate and low electrical activity intervals of ORG rhythms.

Figures 6(c)-6(f) show the values of the features used in the SVM classifier; these values were statistically different for the ORG and shockable rhythms $(P<0.001)$. The SVM based on four features showed a significantly better performance when compared to the SVMs based on the best single, pair, or triplet of features (McNemar's test $\chi^{2}>10, P<0.001$, in all three cases). The optimal working point of the four-feature SVM was $(C=8.5, \gamma=0.1)$, which produced a BER $=0.064$, $\mathrm{TPR}=0.927$, and $\mathrm{TNR}=0.944$ for the SVM classifier. The receiver operating characteristics analysis on the SVM features resulted in the following area under the curve (AUC) values: $0.948,0.928,0.807$, and 0.733 for $b S, n P, P_{\text {fib }}$, and $P_{h}$, respectively. When combined in the SVM the resulting AUC was 0.971 , which reveals the robustness of the classifier.

3.2.2. Test. The optimized SAA was used to classify the $3 \mathrm{~s}$ windows in the test set; Table 2 shows a summary of the results. The overall sensitivity and specificity were $89.7 \%$ (low one-sided 90\% CI, 85.5) and 95.1\% (low 90\% CI, 94.3), respectively. The $9 \mathrm{~s}$ segments were diagnosed using a majority criterion on three consecutive window analyses, this increased the overall sensitivity and specificity to $91.0 \%$ (low 90\% CI, 86.6) and 96.6\% (low 90\% CI, 95.9), respectively, and AHA recommendations were met for all rhythm types (see Table 2).

Figure 7 shows two examples (Figures 7(a) and 7(c)) of correctly diagnosed segments and two examples (Figures 7(b) and $7(d)$ ) of incorrectly diagnosed segments. The examples (Figures $7(\mathrm{a})$ and $7(\mathrm{c})$ ) show that the algorithm works robustly even in the presence of important filtering residuals. However, there were some instances of misdiagnosed segments as shown in Figures 7 (b) and 7(d). Errors were generally caused by spiky filtering residuals in shockable rhythms (Figure 7(b)) or large filtering residuals during ASY (Figure 7(d)).

Processing time for the complete algorithm, CPR suppression filter based on the LMS filter followed by the SAA, was on average $8.7 \mathrm{~ms}$ per $3 \mathrm{~s}$ segment. Processing time was broken down into $5.8 \mathrm{~ms}$ for the LMS filter and $2.9 \mathrm{~ms}$ for the SAA. For decisions taken by the LEA detector the SAA required only $1.8 \mathrm{~ms}$, and for windows in which the LEA detector and the SVM were used it increased to $4.1 \mathrm{~ms}$. In the worst case scenario processing time for the complete algorithm was under $10 \mathrm{~ms}$.

\section{Discussion}

This study presents the first attempt to combine two approaches for rhythm analysis during CPR: adaptive filters 


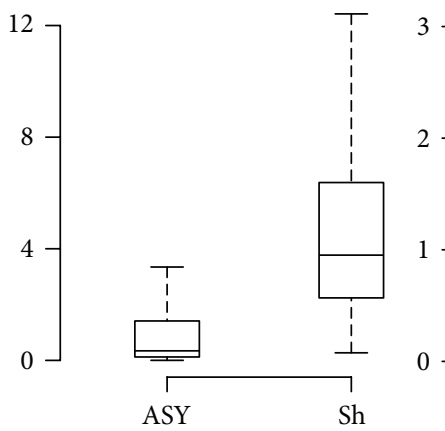

(a) $P_{\mathrm{LEA}}$

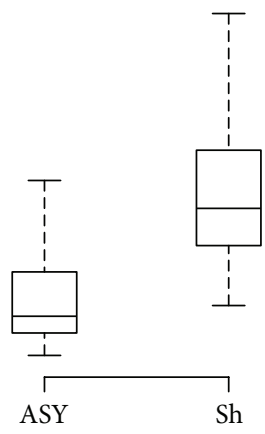

(b) $L_{\min }$

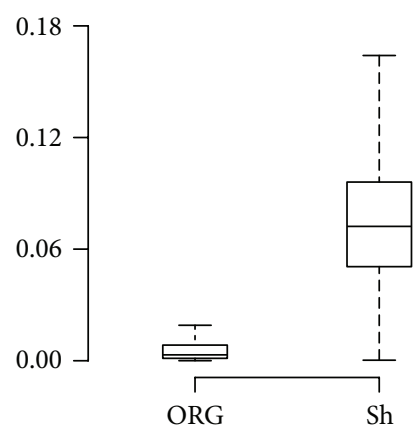

(c) $b S$

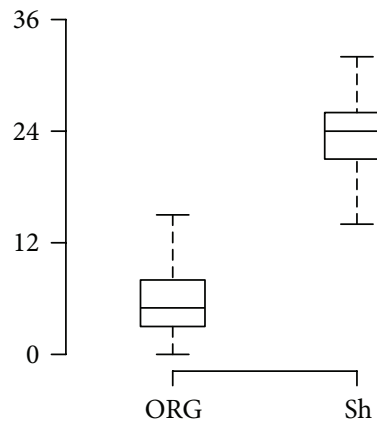

(d) $n P$

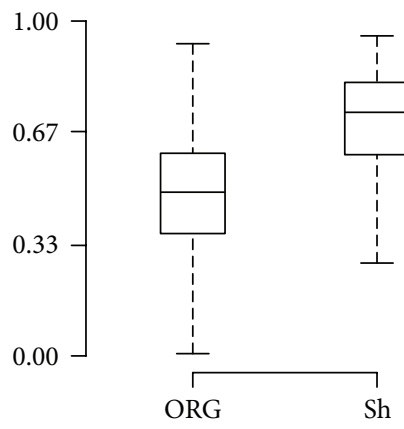

(e) $P_{\text {fib }}$

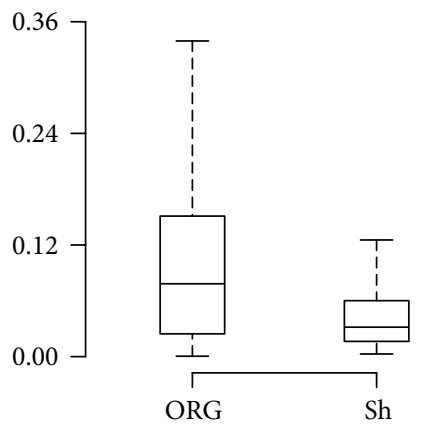

(f) $P_{h}$

FIGURE 6: Features of the SAA for the rhythm types used in each training stage of the algorithm. For the LEA detector the figure compares ASY versus Sh (panels (a) and (b)), and for the SVM classifier ORG versus Sh (panels (c)-(f)). The boxes show the median and interquartile ranges (IQR) and the whisker shows the last datum within the $\pm 1.5 \mathrm{IQR}$ interval. Significant differences were found for the median value of the features between the targeted groups $(P<0.0001)$.

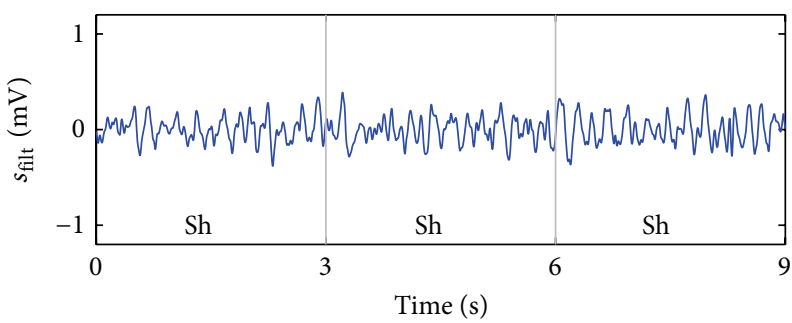

(a) VF

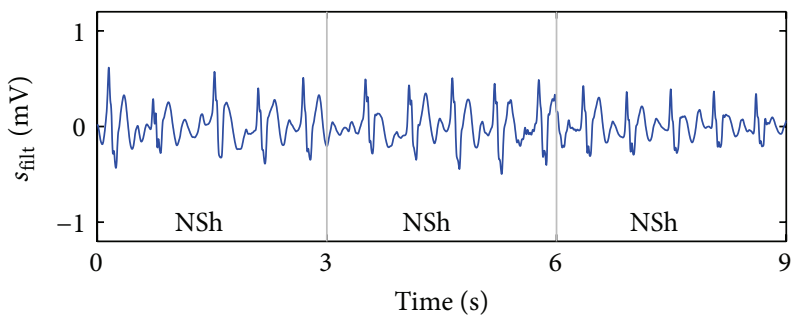

(c) ORG

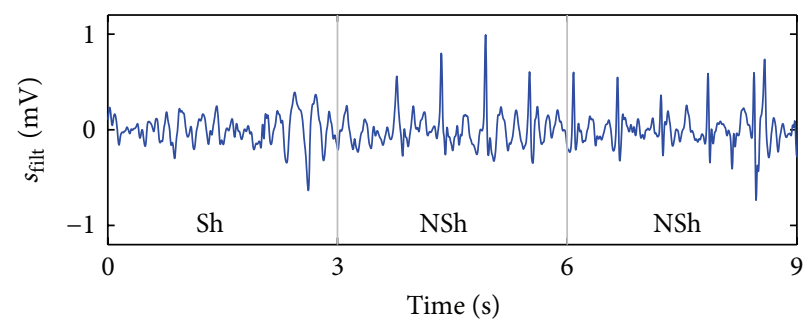

(b) VF

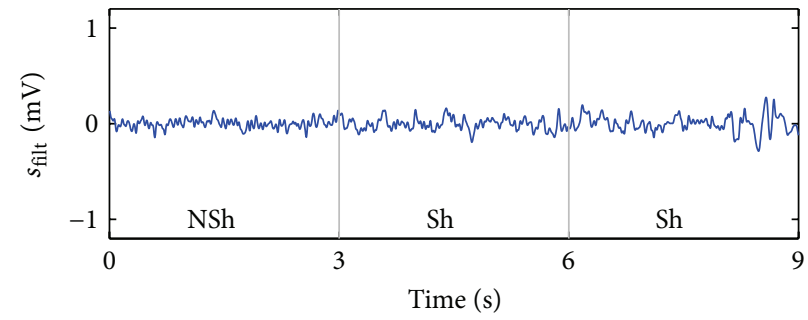

(d) ASY

FiguRE 7: Examples of correctly and incorrectly classified $9 \mathrm{~s}$ segments. Examples $(\mathrm{a}, \mathrm{c})$ are correctly classified despite the presence of large filtering residuals. However, in the VF of panel (b) spiky filtering artifacts cause the erroneous classification. In the ASY of panel (d) filtering residuals are large in the last two windows causing the shock diagnosis. 
TABLE 2: Final classification for the 3-s windows and 9-s segments of the test set compared to the AHA performance goals. Sensitivities, specificities and low one-sided 90\% CIs (in parenthesis) were obtained using GEE to adjust for clustering.

\begin{tabular}{|c|c|c|c|c|c|}
\hline \multirow{2}{*}{ Rhythm type } & \multicolumn{2}{|c|}{ 3-s window } & \multicolumn{2}{|c|}{ 9-s segment } & \multirow{2}{*}{ AHA goal [18] } \\
\hline & $n$ & $\mathrm{Se} / \mathrm{Sp}$ & $n$ & $\mathrm{Se} / \mathrm{Sp}$ & \\
\hline Shockable & 1866 & $89.7(85.5)$ & 622 & $91.0(86.6)$ & $>90$ (for VF) \\
\hline Nonshockable & 10050 & $95.1(94.3)$ & 3350 & $96.6(95.9)$ & $>95$ \\
\hline AS & 3927 & $94.3(93.1)$ & 1309 & $96.5(95.2)$ & $>95$ \\
\hline ORG & 6123 & $95.6(94.6)$ & 2041 & $96.7(95.8)$ & $>95$ \\
\hline
\end{tabular}

TABLE 3: Comparative assessment in terms of accuracy and the composition of the databases (\% of ASY in nonshockable rhythm in parenthesis) between the method proposed in this study and previous methods tested on OHCA rhythms.

\begin{tabular}{|c|c|c|c|c|c|}
\hline \multirow{2}{*}{ Authors } & \multirow{2}{*}{ Method } & \multicolumn{2}{|c|}{ Accuracy } & \multicolumn{2}{|c|}{ Testing datasets } \\
\hline & & $\mathrm{Se}(\%)$ & $\mathrm{Sp}(\%)$ & Sh & NSh \\
\hline Eilevstjønn et al. [10] & MC-RAMP & 96.7 & 79.9 & 92 & $174(30 \%)$ \\
\hline Aramendi et al. [19] & LMS filter & 95.4 & 86.3 & 87 & $285(31 \%)$ \\
\hline Tan et al. [20] & ART filter & 92.1 & 90.5 & 114 & 4155 (NA) \\
\hline Li et al. [5] & Direct analysis & 93.3 & 88.6 & 1256 & $964(4 \%)$ \\
\hline Krasteva et al. [6] & Direct analysis & 90.1 & 86.1 & 172 & $721(46 \%)$ \\
\hline Proposed method & Filtering + SAA & 91.0 & 96.6 & 622 & $3350(39 \%)$ \\
\hline
\end{tabular}

to suppress the CPR artifact and an SAA optimized to analyze the rhythm after filtering. Our objective was to increase the specificity, because the low specificity of current methods has restrained their implementation in current defibrillators. Our results indicate that our new design approach might contribute to a substantial increase of the accuracy of rhythm analysis methods during CPR, with results that marginally meet AHA performance goals.

The design efforts were focused on obtaining a high specificity during CPR to allow CCs to continue uninterrupted until the method gives a shock advice. The positive predictive value (PPV) of the algorithm, that is, the confidence in a shock diagnosis, must be kept high to avoid unnecessary CPR interruptions if the underlying rhythm is nonshockable. Since VF is the positive class, the PPV depends on the sensitivity/specificity of the algorithm and on the prevalence of VF, $P_{\mathrm{vf}}$, in the following way:

$$
\begin{aligned}
\operatorname{PPV}(\%) & =100 \times \frac{\mathrm{TP}}{\mathrm{TP}+\mathrm{FP}} \\
& =100 \times \frac{\mathrm{Se} \cdot P_{\mathrm{vf}}}{\mathrm{Se} \cdot P_{\mathrm{vf}}+(1-\mathrm{Sp}) \cdot\left(1-P_{\mathrm{vf}}\right)} .
\end{aligned}
$$

The exact prevalence of VF (reported for the initially observed rhythm as stated in [33]) is unknown and varies among OHCA studies, with figures in the range of $23 \%$ to $67 \%[34,35]$. For the original OHCA studies from which our datasets originated the prevalences of VF were $43 \%$ [21] and $41 \%$ [22], within the previous range. For the limits of the VF prevalence range, the PPV of our algorithm is high, in the $88.9 \%$ to $98.2 \%$ range. Furthermore, since the PPV depends on the prevalences, algorithms must be trained to optimize sensitivity/specificity, with emphasis on a large specificity (a specificity of $100 \%$ would result in a PPV of $100 \%$ regardless of the prevalences).
To this date most methods for rhythm analysis during CPR have focused on the accurate detection of shockable rhythms, resulting in higher values for sensitivity than for specificity. Table 3 compares the accuracy of our method to that of five well-known methods tested on OHCA data that represent the two most successful strategies for rhythm analysis during CPR. Three of those methods are based on adaptive filters $[10,12,20]$, and the other two are algorithms designed to directly diagnose the corrupt ECG $[5,6]$. Although the sensitivity of our method is up to 4 points below that reported by methods based on adaptive filters, it is still above the value recommended by the AHA, which ensures the detection of a high proportion of shockable rhythms. The higher sensitivity of methods based on adaptive filters may be explained by the fact that filtering residuals are frequently diagnosed as shockable by SAA designed to diagnose artifact-free ECG [14]. In contrast, the $96.6 \%$ specificity of our approach is an important improvement with respect to previous approaches in which the specificity was below $91 \%$. We showed that combining the strong points of both approaches may result in an increased accuracy.

The characteristics of the OHCA data used in these studies may affect the sensitivity/specificity results, and in particular the characteristics of $\mathrm{CPR}$, the selection criteria for VF, and the proportion of ASY among nonshockable rhythms. Rate and depth values of CPR in our data are similar to those reported in the original studies [21, 22] and represent the wide range of $\mathrm{CPR}$ characteristics found in the field. In particular, the CC rates are high (around $120 \mathrm{cpm}$ ), the spectral overlap with OHCA rhythms is therefore large, and suppressing the CPR artifact in our data should be challenging [8]. The CC depth was low even according to the 2000 resuscitation guidelines and lower than the $5 \mathrm{~cm}$ recommended in current guidelines [36]. However, no clear 
correlation between depth and larger artifacts has been demonstrated to date on human data. Our database only included VF annotated as coarse, as stated in the AHA statement. The three-phase model of cardiac arrest suggests that fine VF occurs when VF transitions from the electric phase into the circulatory or metabolic phases [37]. There is no conclusive evidence that immediate defibrillation is the optimal treatment in these latter phases of VF [38], so from a SAA design perspective it is a sound decision to only include coarse VF. On the other hand, our database has a large proportion of ASY among nonshockable rhythms (39\%), in agreement with the fact that ASY is the most frequent nonshockable OHCA rhythm [39]. The high specificity of our method for ASY is particularly important because ASY is the most difficult nonshockable rhythm to detect during CPR $[14,16]$.

Our study shows that combining adaptive filtering with special SAAs that optimally diagnose the filtered ECG may result in an increased overall accuracy. In addition, the computational cost of the algorithm is low, as shown by the processing time analysis. The SAA algorithm computes at most six ECG features, and implementing our SVM in an AED requires only a few kilobytes of memory for the support vectors and the computation of the discriminant function (see equation (5)). The LMS algorithm using 5 harmonics involves only 10 coefficients [12], which substantially simplifies the filter. In any case, incorporating a CPR artifact filter to current AEDs is more complex than using algorithms that directly analyze the corrupt ECG $[5,6]$. Filtering techniques based on the $C D$ signal require the use of external CPR quality devices $[40,41]$ or modified defibrillation pads [42, 43] to record the acceleration signal. Alternatively other reference signals can be used, such as the thoracic impedance recorded through the defibrillation pads [19]. CPR artifact filters increase the complexity of the software and signal processing units of the AED and may even demand changes in its hardware to acquire reference signals.

Finally, several studies need to be completed before any method could be safely taken to the field. First, more conclusive results require testing the algorithm on data recorded by equipment different from those used for this study and with CPR delivered according to the latest 2010 CPR guidelines. In addition, retrospective studies based on complete resuscitation episodes should be conducted. In this way, the impact of using the method on CPR administration could be evaluated. This involves, among other things, a statistical evaluation of whether the method avoids unnecessary CPR interruptions in nonshockable rhythms and unnecessary CPR prolongations in shockable rhythms [36]. The methodology for such an evaluation has recently been developed [44].

\section{Conclusions}

This work introduces a new method for rhythm analysis during CPR with a novel design approach aimed at obtaining a high specificity. The method combines an adaptive LMS filter to suppress the CPR artifact with a new shock/noshock classification method based on the analysis of the filtered ECG. The method resulted in an increased specificity of $96.6 \%$ without compromising the sensitivity, with overall performance figures that met AHA requirements.

\section{Conflict of Interests}

The authors declare that there is no conflict of interests regarding the publication of this paper.

\section{Acknowledgments}

This work received financial support from Spanish Ministerio de Economía y Competitividad (Projects TEC2012-31144 and TEC2012-31928), from the UPV/EHU (unit UFI11/16), and from the Basque government (Grants BFI-2010-174, BFI2010-235, and BFI-2011-166). The authors would like to thank Professor Rojo-Álvarez from the University Rey Juan Carlos (Madrid, Spain) for his assistance with SVM classifiers and for his thorough review of the paper.

\section{References}

[1] J. Berdowski, R. A. Berg, J. G. P. Tijssen, and R. W. Koster, "Global incidences of out-of-hospital cardiac arrest and survival rates: systematic review of 67 prospective studies," Resuscitation, vol. 81, no. 11, pp. 1479-1487, 2010.

[2] R. O. Cummins, J. P. Ornato, W. H. Thies et al., "Improving survival from sudden cardiac arrest: the "chain of survival" concept," Circulation, vol. 83, no. 5, pp. 1832-1847, 1991.

[3] C. D. Deakin, J. P. Nolan, J. Soar et al., "European Resuscitation Council Guidelines for resuscitation 2010 section 4. Adult advanced life support," Resuscitation, vol. 81, no. 10, pp. 13051352, 2010.

[4] S. Cheskes, R. H. Schmicker, J. Christenson et al., "Perishock pause: an independent predictor of survival from out-ofhospital shockable cardiac arrest," Circulation, vol. 124, no. 1, pp. 58-66, 2011.

[5] Y. Li, J. Bisera, F. Geheb, W. Tang, and M. H. Weil, "Identifying potentially shockable rhythms without interrupting cardiopulmonary resuscitation," Critical Care Medicine, vol. 36, no. 1, pp. 198-203, 2008.

[6] V. Krasteva, I. Jekova, I. Dotsinsky, and J.-P. Didon, "Shock advisory system for heart rhythm analysis during cardiopulmonary resuscitation using a single ecg input of automated external defibrillators," Annals of Biomedical Engineering, vol. 38, no. 4, pp. 1326-1336, 2010.

[7] S. Ruiz de Gauna, U. Irusta, J. Ruiz, U. Ayala, E. Aramendi, and T. Eftestøl, "Rhythm analysis during cardiopulmonary resuscitation: past, present, and future," BioMed Research International, vol. 2014, Article ID 386010, 13 pages, 2014.

[8] T. Werther, A. Klotz, M. Granegger et al., "Strong corruption of electrocardiograms caused by cardiopulmonary resuscitation reduces efficiency of two-channel methods for removing motion artefacts in non-shockable rhythms," Resuscitation, vol. 80, no. 11, pp. 1301-1307, 2009. 
[9] S. O. Aase, T. Eftestøl, J. H. Husøy, K. Sunde, and P. A. Steen, "CPR artifact removal from human ECG using optimal multichannel filtering," IEEE Transactions on Biomedical Engineering, vol. 47, no. 11, pp. 1440-1449, 2000.

[10] J. Eilevstjønn, T. Eftestøl, S. O. Aase, H. Myklebust, J. H. Husøy, and P. A. Steen, "Feasibility of shock advice analysis during CPR through removal of CPR artefacts from the human ECG," Resuscitation, vol. 61, no. 2, pp. 131-141, 2004.

[11] R. D. Berger, J. Palazzolo, and H. Halperin, "Rhythm discrimination during uninterrupted CPR using motion artifact reduction system," Resuscitation, vol. 75, no. 1, pp. 145-152, 2007.

[12] U. Irusta, J. Ruiz, S. R. de Gauna, T. Eftestøl, and J. KramerJohansen, "A least mean-Square filter for the estimation of the cardiopulmonary resuscitation artifact based on the frequency of the compressions," IEEE Transactions on Biomedical Engineering, vol. 56, no. 4, pp. 1052-1062, 2009.

[13] K. Rheinberger, T. Steinberger, K. Unterkofler, M. Baubin, A. Klotz, and A. Amann, "Removal of CPR artifacts from the ventricular fibrillation ECG by adaptive regression on lagged reference signals," IEEE Transactions on Biomedical Engineering, vol. 55, no. 1, pp. 130-137, 2008.

[14] J. Ruiz, U. Irusta, S. Ruiz de Gauna, and T. Eftestøl, "Cardiopulmonary resuscitation artefact suppression using a Kalman filter and the frequency of chest compressions as the reference signal," Resuscitation, vol. 81, no. 9, pp. 1087-1094, 2010.

[15] E. Aramendi, S. R. de Gauna, U. Irusta, J. Ruiz, M. F. Arcocha, and J. M. Ormaetxe, "Detection of ventricular fibrillation in the presence of cardiopulmonary resuscitation artefacts," Resuscitation, vol. 72, no. 1, pp. 115-123, 2007.

[16] S. Ruiz de Gauna, J. Ruiz, U. Irusta, E. Aramendi, T. Eftestøl, and J. Kramer-Johansen, "A method to remove CPR artefacts from human ECG using only the recorded ECG," Resuscitation, vol. 76, no. 2, pp. 271-278, 2008.

[17] Y. Li and W. Tang, "Techniques for artefact filtering from chest compression corrupted ECG signals: good, but not enough," Resuscitation, vol. 80, no. 11, pp. 1219-1220, 2009.

[18] R. E. Kerber, L. B. Becker, J. D. Bourland et al., "Automatic external defibrillators for public access defibrillation: recommendations for specifying and reporting arrhythmia analysis algorithm performance, incorporating new waveforms, and enhancing safety," Circulation, vol. 95, no. 6, pp. 1677-1682, 1997.

[19] E. Aramendi, U. Ayala, U. Irusta, E. Alonso, T. Eftestøl, and J. Kramer-Johansen, "Suppression of the cardiopulmonary resuscitation artefacts using the instantaneous chest compression rate extracted from the thoracic impedance," Resuscitation, vol. 83, no. 6, pp. 692-698, 2012.

[20] Q. Tan, G. A. Freeman, F. Geheb, and J. Bisera, "Electrocardiographic analysis during uninterrupted cardiopulmonary resuscitation," Critical Care Medicine, vol. 36, no. 11, pp. S409-S412, 2008.

[21] L. Wik, J. Kramer-Johansen, H. Myklebust et al., "Quality of cardiopulmonary resuscitation during out-of-hospital cardiac arrest," Journal of the American Medical Association, vol. 293, no. 3, pp. 299-304, 2005.

[22] J. Kramer-Johansen, H. Myklebust, L. Wik et al., "Quality of out-of-hospital cardiopulmonary resuscitation with real time automated feedback: a prospective interventional study," Resuscitation, vol. 71, no. 3, pp. 283-292, 2006.

[23] D. L. Atkins, W. A. Scott, A. D. Blaufox et al., "Sensitivity and specificity of an automated external defibrillator algorithm designed for pediatric patients," Resuscitation, vol. 76, no. 2, pp. 168-174, 2008.
[24] A. C. Clifford, "Comparative assessment of shockable ECG rhythm detection algorithms in automated external defibrillators," Resuscitation, vol. 32, no. 3, pp. 217-225, 1996.

[25] W. Zong, G. B. Moody, and D. Jiang, "A robust open-source algorithm to detect onset and duration of QRS complexes," in Proceedings of the IEEE Computers in Cardiology, pp. 737-740, September 2003.

[26] C. G. Brown and R. Ozwonczyk, "Signal analysis of the human electrocardiogram during ventricular fibrillation: frequency and amplitude parameters as predictors of successful countershock," Annals of Emergency Medicine, vol. 27, no. 2, pp. 184-188, 1996.

[27] K. Minami, H. Nakajima, and T. Toyoshima, "Real-time discrimination of ventricular tachyarrhythmia with Fourier-transform neural network," IEEE Transactions on Biomedical Engineering, vol. 46, no. 2, pp. 179-185, 1999.

[28] A. Ben-Hur and J. Weston, “A user's guide to support vector machines," Data Mining Techniques for the Life Sciences, vol. 609, pp. 223-239, 2010.

[29] C.-C. Chang and C.-J. Lin, "LIBSVM: a Library for support vector machines," ACM Transactions on Intelligent Systems and Technology, vol. 2, no. 3, article 27, 2011.

[30] S. L. Zeger and K. Y. Liang, "Longitudinal data analysis for discrete and continuous outcomes," Biometrics, vol. 42, no. 1, pp. 121-130, 1986.

[31] M. R. Sternberg and A. Hadgu, "A GEE approach to estimating sensitivity and specificity and coverage properties of the confidence intervals," Statistics in Medicine, vol. 20, no. 9-10, pp. 1529-1539, 2001.

[32] U. Halekoh, S. Højsgaard, and J. Yan, "The R package geepack for generalized estimating equations," Journal of Statistical Software, vol. 15, no. 2, pp. 1-11, 2006.

[33] I. Jacobs, V. Nadkarni, J. Bahr et al., "Cardiac arrest and cardiopulmonary resuscitation outcome reports: update and simplification of the Utstein templates for resuscitation registries. A statement for healthcare professionals from a task force of the international liaison committee on resuscitation (American Heart Association, European Resuscitation Council, Australian Resuscitation Council, New Zealand Resuscitation Council, Heart and Stroke Foundation of Canada, InterAmerican Heart Foundation, Resuscitation Council of Southern Africa)," Resuscitation, vol. 63, no. 3, pp. 233-249, 2004.

[34] C. Atwood, M. S. Eisenberg, J. Herlitz, and T. D. Rea, "Incidence of EMS-treated out-of-hospital cardiac arrest in Europe," Resuscitation, vol. 67, no. 1, pp. 75-80, 2005.

[35] G. Nichol, E. Thomas, C. W. Callaway et al., "Regional variation in out-of-hospital cardiac arrest incidence and outcome," Journal of the American Medical Association, vol. 300, no. 12, pp. 1423-1431, 2008.

[36] M. R. Sayre, R. W. Koster, M. Botha et al., "Part 5: Adult basic life support: 2010 International Consensus on Cardiopulmonary Resuscitation and Emergency Cardiovascular Care Science with Treatment Recommendations," Circulation, vol. 122, no. 16, supplement 2, pp. S298-S324, 2010.

[37] M. L. Weisfeldt and L. B. Becker, "Resuscitation after cardiac arrest: a 3-phase time-sensitive model," Journal of the American Medical Association, vol. 288, no. 23, pp. 3035-3038, 2002.

[38] J. P. Freese, D. B. Jorgenson, P. Y. Liu et al., "Waveform analysisguided treatment versus a standard shock-first protocol for the treatment of out-of-hospital cardiac arrest presenting in ventricular fibrillation: results of an international randomized, controlled trial," Circulation, vol. 128, no. 9, pp. 995-1002, 2013. 
[39] L. A. Cobb, C. E. Fahrenbruch, M. Olsufka, and M. K. Copass, "Changing incidence of out-of-hospital ventricular fibrillation, 1980-2000," Journal of the American Medical Association, vol. 288, no. 23, pp. 3008-3013, 2002.

[40] C. N. Pozner, A. Almozlino, J. Elmer, S. Poole, D. McNamara, and D. Barash, "Cardiopulmonary resuscitation feedback improves the quality of chest compression provided by hospital health care professionals," American Journal of Emergency Medicine, vol. 29, no. 6, pp. 618-625, 2011.

[41] G. D. Perkins, R. P. Davies, S. Quinton et al., "The effect of real-time CPR feedback and post event debriefing on patient and processes focused outcomes: a cohort study: trial protocol," Scandinavian Journal of Trauma, Resuscitation and Emergency Medicine, vol. 19, article 58, 2011.

[42] C. F. Babbs, A. E. Kemeny, W. Quan, and G. Freeman, "A new paradigm for human resuscitation research using intelligent devices," Resuscitation, vol. 77, no. 3, pp. 306-315, 2008.

[43] K. G. Monsieurs, M. de Regge, K. Vansteelandt et al., "Excessive chest compression rate is associated with insufficient compression depth in prehospital cardiac arrest," Resuscitation, vol. 83, no. 11, pp. 1319-1323, 2012.

[44] J. Ruiz, U. Ayala, S. Ruiz de Gauna et al., "Direct evaluation of the effect of filtering the chest compression artifacts on the uninterrupted cardiopulmonary resuscitation time," American Journal of Emergency Medicine, vol. 31, no. 6, pp. 910-915, 2013. 


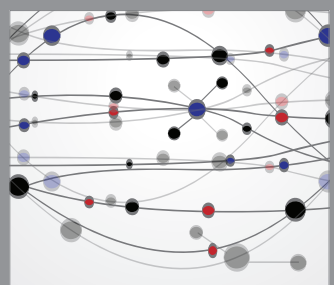

The Scientific World Journal
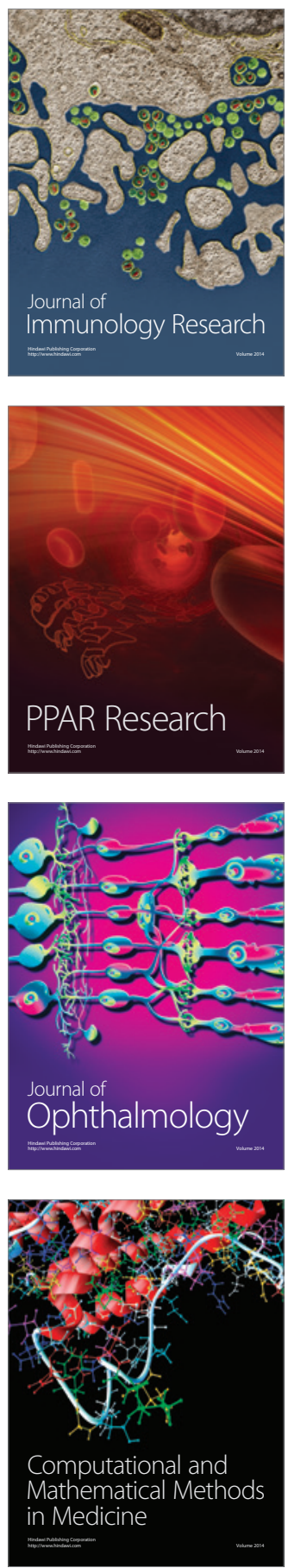

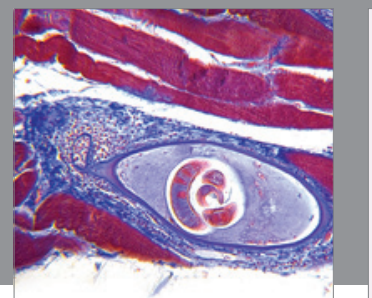

Gastroenterology

Research and Practice
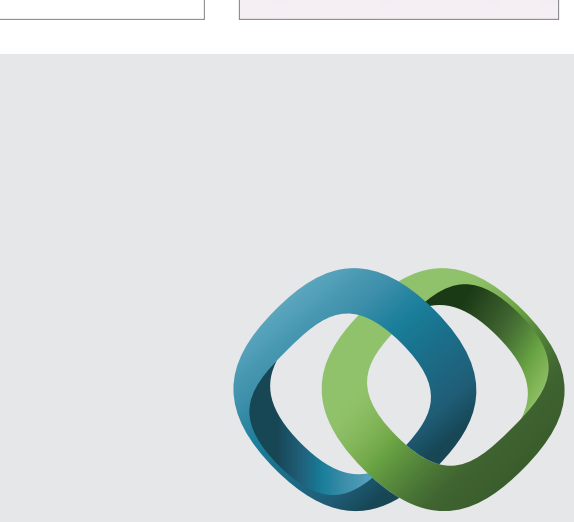

\section{Hindawi}

Submit your manuscripts at

http://www.hindawi.com
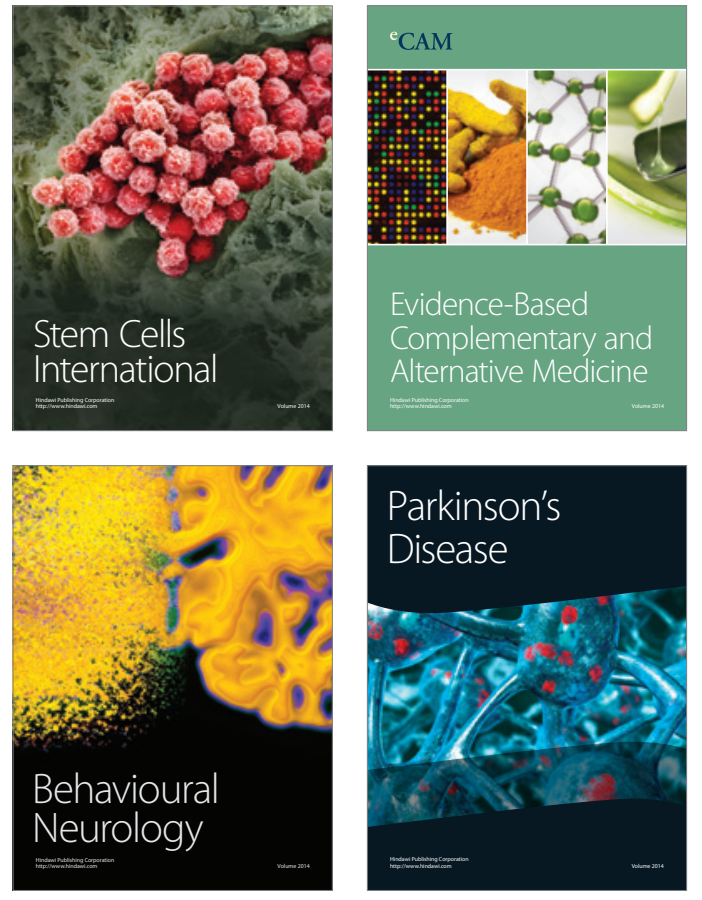
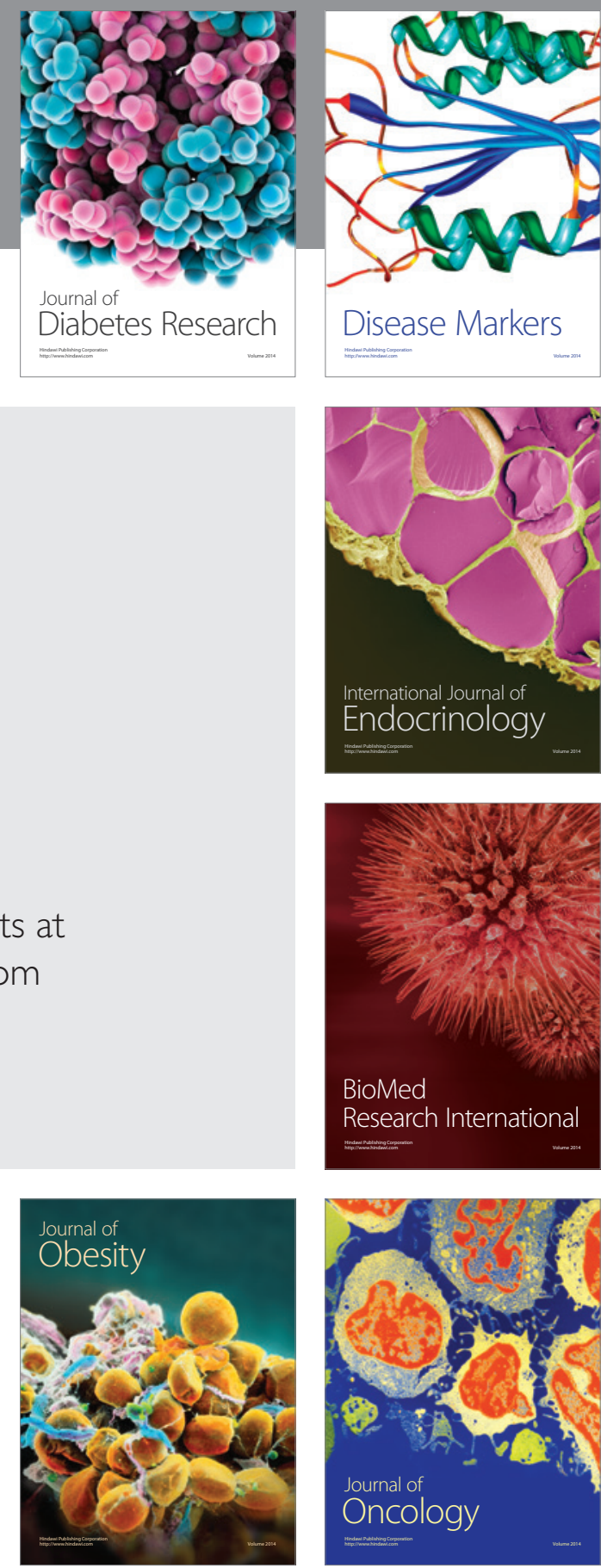

Disease Markers
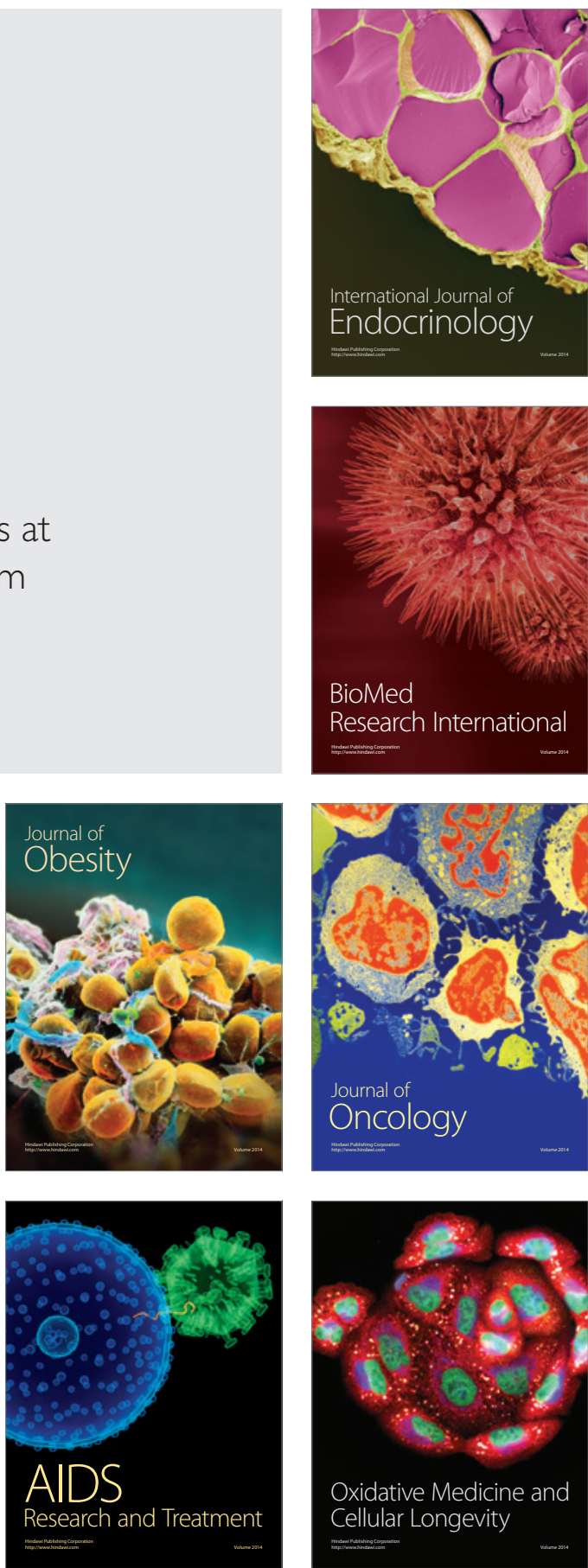